\title{
The sharpest view of the local AGN population at mid-infrared wavelengths
}

\author{
Daniel Asmus, ${ }^{1,2}$ Sebastian F. Hönig, ${ }^{3,2,4}$ Poshak Gandhi, ${ }^{5,6}$ \\ Alain Smette ${ }^{7}$ and Wolfgang J. Duschl ${ }^{2,8}$ \\ ${ }^{1}$ Max-Planck-Institut für Radioastronomie, Auf dem Hügel 69, 53121 Bonn, Germany \\ email: asmus@mpifr.de \\ ${ }^{2}$ Institut für Theoretische Physik und Astrophysik, Christian- Albrechts-Universität zu Kiel, \\ Leibnizstr. 15, 24098 Kiel, Germany \\ ${ }^{3}$ Dark Cosmology Center, Niels Bohr Institute, University of Copenhagen, Juliane Maries Vej \\ 30, 2100 Copenhagen, Denmark \\ ${ }^{4}$ UCSB Department of Physics, Broida Hall 93106-9530, Santa Barbara, CA, USA \\ ${ }^{5}$ Department of Physics, Durham University, South Road, Durham, DH1 3LE, United \\ Kingdom \\ ${ }^{6}$ Institute of Space and Astronautical Science (ISAS), Japan, Aerospace Exploration Agency, \\ 3-1-1 Yoshinodai, chuo-ku, \\ Sagamihara, Kanagawa 252-5210, Japan \\ ${ }^{7}$ European Southern Observatory, Casilla 19001, Santiago 19, Chile \\ ${ }^{8}$ Steward Observatory, The University of Arizona, 933 N. Cherry Ave, Tucson, AZ 85721, USA
}

\begin{abstract}
We present the largest mid-infrared (MIR) atlas of active galactic nuclei at sub-arcsec spatial scales containing 253 objects with a median redshift of 0.016 . It comprises all available ground-based high-angular resolution MIR observations performed to date with 8-meter class telescopes and includes 895 photometric measurements. All types of AGN are present in the atlas, which also includes 80 per cent of the 9-month BAT AGN sample. Therefore, this atlas and its subsamples are very well-suited for AGN unification studies. A first application of the atlas is the extension of the MIR-X-ray luminosity correlation for AGN.
\end{abstract}

Keywords. galaxies: active - galaxies: nuclei - infrared: galaxies

\section{The AGN mid-infrared atlas}

The Sub-arcsecond mid-infrared (MIR) atlas of local active galactic nuclei (AGN) is a collection of all available $N$ - and $Q$-band images obtained at ground-based 8-meter class telescopes with public archives (Gemini/Michelle, Gemini/T-ReCS, Subaru/COMICS, and VLT/VISIR). The superb angular resolution isolates the AGN from the host emission and makes the atlas a powerful tool for AGN unification studies. It includes 253 local AGN with a median redshift of 0.016 captured in 895 images in various filters between $\sim 7$ to $13 \mu \mathrm{m}$ and $\sim 18 \mu \mathrm{m}$, of which $60 \%$ are previously unpublished. These and the corresponding nuclear photometry will appear in Asmus et al. (2014a). The photometry and reduced images will also be accessible online through the VO and CDS. Out of the 253 observed objects, 208 were detected (Fig. 1). Extended nuclear emission is present in at least $21 \%$ of the objects, while elongated nuclear emission usually aligns with the ionization cones in Seyfert type objects.

Finally, preliminary analysis shows a strong correlation between the nuclear MIR and the absorption-corrected hard X-ray luminosities, the latter ones collected from the literature. There are no significant differences between the AGN types or trends with the source luminosity (see Asmus et al. 2014b). 


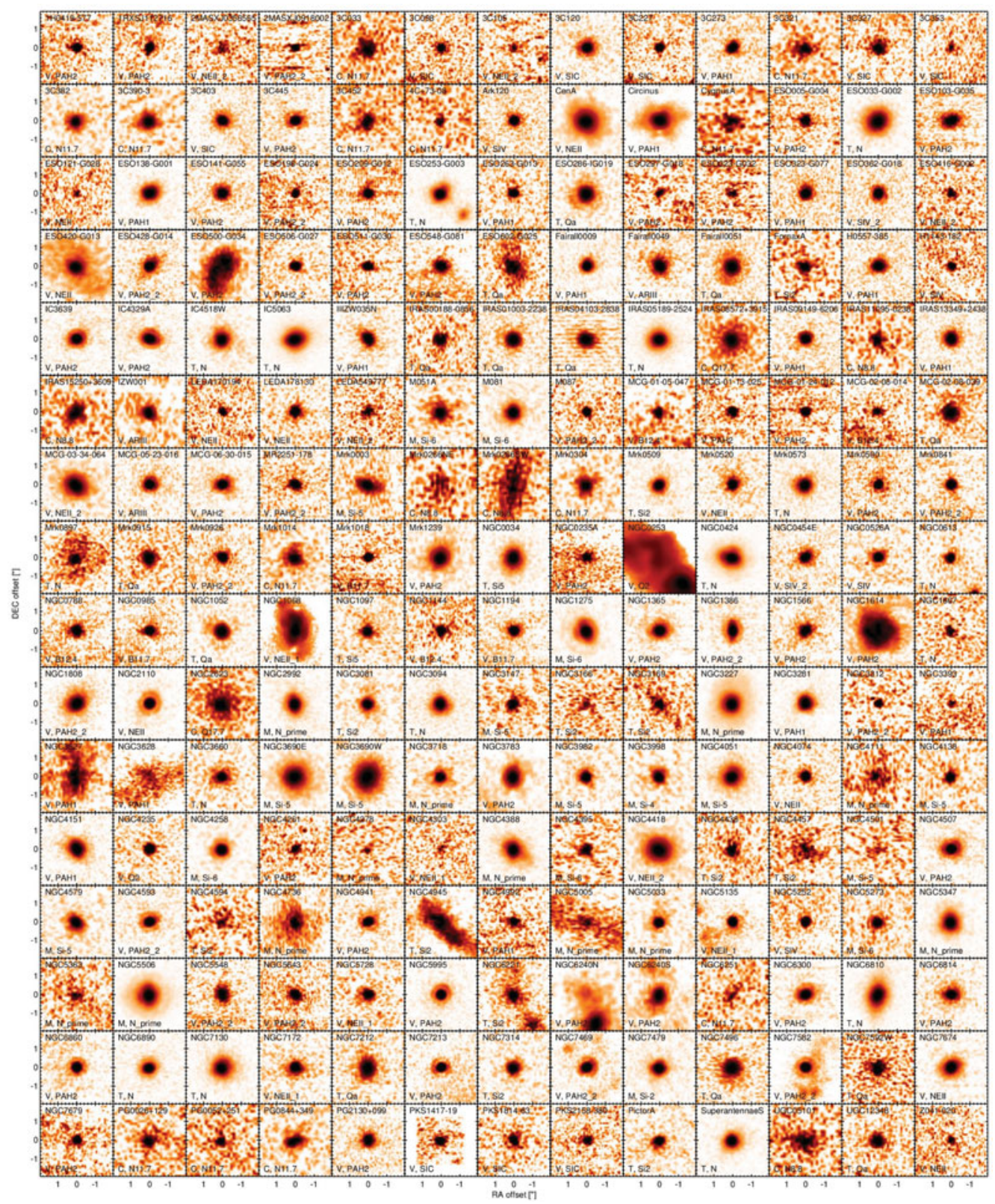

Figure 1. Subarcsecond-resolution MIR images of all objects with detected emission. For each object only the image with the highest-S/N is shown. Displayed are the inner 4" with North up and East to the left. The colour scaling is logarithmic with white corresponding to median background and black to the $1 \%$ of pixels with the highest flux values. Reproduced from Asmus et al. (2014a).

\section{References}

Asmus, D., Hönig, S. F., Gandhi, P., Smette, A., \& Duschl, W. J. 2014, MNRAS, 439, 1648 Asmus, D., Gandhi, P., Hönig, S. F., Smette, A., \& Duschl, W. J. 2014, MNRAS, in prep. 